\title{
Spermatic vein embolization as a treatment for symptomatic varicocele
}

Mark P. Broe ${ }^{1}$; James P.C. Ryan; Eanna J. Ryan; David J. Murphy²; David W. Mulvin $^{1}$; Colin Cantwell2; David P. Brophy ${ }^{2}$

${ }^{1}$ Deptartment of Urology, St. Vincent's University Hospital, Dublin, Ireland; ${ }^{2}$ Department of Radiology, St. Vincent's University Hospital, Dublin, Ireland

Cite as: Broe MP, Ryan JPC, Ryan EJ, et al. Spermatic vein embolization as a treatment for symptomatic varicocele. Can Urol Assoc J 2021 May 11; Epub ahead of print. http://dx.doi.org/10.5489/cuaj.7077

Published online May 11, 2021

Correspondence: Dr. Mark P. Broe, St. Vincent's University Hospital, Dublin, Ireland; markbroe@rcsi.ie

$* * *$

\section{Abstract}

Introduction: Varicocele is a relatively common condition in men that causes pain in approximately $10 \%$ of cases. There have been few studies to date assessing the improvements in both pain and quality of life parameters associated with spermatic vein embolization (SVE) as a treatment for patients with symptomatic varicocele, so we aimed to assess this.

Methods: A review was carried out of consecutive SVE procedures performed at our institution from 2013-2019. Only patients with painful varicocele were included after other causes of testicular pain were excluded. The technique employed was a combination of distal coil embolization of the spermatic vein with 4-6 $\mathrm{mm}$ coils at the level of the inguinal canal, as well as sclerotherapy to prevent reflux of sclerosant. Furthermore, a prospective validated Pain Impact Questionnaire-6 (PIQ-6) was performed to assess for improvement in quality of life. A matched pair Student twotailed t-test was used to compare mean scores pre- and post-treatment, with $95 \%$ confidence intervals presented as $\mathrm{T}$ scores and their associated $\mathrm{p}$-values.

Results: Over six years, 62 SVE procedures were performed for symptomatic varicocele. Success rate was $95 \%$, with a median follow up of nine months. Two patients had a failed procedure on two occasions requiring subsequent surgical ligation. There was one clinically significant recurrence. All components of PIQ-6 score showed a statistically significant reduction post-SVE, most noticeably pain severity and impact on leisure activities.

Conclusions: SVE is a safe, effective, and well-tolerated treatment for symptomatic varicocele, improving pain and quality of life. 


\section{Introduction}

The incidence of varicocele in young healthy men is $8-23 \%{ }^{1}$. Traditionally treatment was in the form of open surgical ligation, with high retroperitoneal, inguinal and sub-inguinal techniques all described. Laparoscopic and particularly microsurgical techniques have improved outcomes and post-operative recovery for patients undergoing this procedure ${ }^{2}$. Regardless of surgical approach there remains a considerable recurrence rate ranging between $3.9-17 \%{ }^{3}$. Spermatic vein embolisation (SVE) was first described in $1979^{4}$. Initially it was employed mainly in the setting of recurrence ${ }^{5}$. However, it has become a common primary modality of treatment in recent years due to a high success rate and low complication rate, particularly with regards to hydrocoele formation ${ }^{6}$.

The indications for treating a varicocele are pain, very large size, young age with reduced testicular volume and subfertility with altered semen parameters and no other cause found. By far the most extensive research into the treatment of varicoceles in the setting of infertility for the improvement of semen parameters. There has been conflicting and controversial evidence on fertility rates ${ }^{78}$. This is reflected in differing guideline recommendations by region ${ }^{9}{ }^{10}$. On the contrary testicular pain, seen in approximately $10 \%$ of patients ${ }^{11} 12$, can be overlooked as the primary intention of treating this condition. Most commonly the pain takes the form of dragging sensation or rarely a more severe intermittent sharp pain. Testicular pain can have a significant impact on a man's quality of life ${ }^{13}$ and should not be overlooked. The treatment of any reversible cause of testicular pain should be a priority in order to avoid development of chronic orchialgia/chronic pelvic pain syndrome and the difficulties associated with management of such a condition ${ }^{14}$.

Whilst exposure to varicocele embolization in radiology training in the past was only in select pioneering centres, the procedure is now established and forms part of the curriculum for produced by the Cardiovascular and Interventional Radiological Society of Europe (CIRSE) and is examined in the accredited European Board of Interventional Radiology (EBIR) examination. It is listed amongst the vascular procedures recommended for higher radiology trainees to be exposed during their training in Ireland ${ }^{15}$. SVE is now offered in most tertiary referral hospitals. With the availability of this procedure at our institution the aim of the current study was to assess the outcomes of consecutive patients who underwent SVE for painful varicocele.

\section{Methods}

\section{Assessment and inclusion criteria}

A prospective study was carried out of consecutive SVEs performed for management of painful varicocele from January 2013- August 2019 at our institution. All patients were assessed by a urologist with history and clinical examination, both sitting and standing and including Valsalva technique. Clinical 
grading system used was that described by Dubin and Amelar ${ }^{16}$. Furthermore, patients underwent doppler ultrasound of testes using a $10 \mathrm{~Hz}$ probe. The size of varicocele on ultrasound was reported as small/mild, medium/moderate or large/severe. Only consenting patients undergoing SVE for management of painful varicocele were included. Exclusion criteria were SVE performed for infertility and patients who had other causes of scrotal pain, such as chronic epididymitis, post inguinal hernia repair or post vasectomy pain syndrome. Patients with subclinical varicocele on ultrasound were not offered intervention. There were two main elements to the study, looking at the technical success and complications of the procedure and also the quality-of-life impact for the patient

\section{Procedure}

All embolisation procedures were performed under conscious sedation (fentanyl and midazolam) as a day case procedure. Each SVE procedure was performed by one of two fellowship trained interventional radiologists (DPB and CC). Pre-procedural antibiotics were not administered. Following central venous access, via a right common femoral vein puncture under ultrasound and fluoroscopic guidance a 5French (Fr) sheath was placed. The testicular vein was accessed using a hydrophilic guidewire and varying combinations of Terumo Medical Cobra 2 (C2) and Simmons 1 (Sim 1) GLIDECATH ${ }^{\circledR}$ peripheral hydrophilic coated catheters for selectivity and navigation. A push venogram was performed point to confirm position in the testicular vein using iodinated contrast. The spermatic vein was accessed with a 5-Fr single end-hole catheter or a microcatheter. Distal coil embolisation was performed of the spermatic vein with coils (usually two $6 \mathrm{~mm}$ and one $4 \mathrm{~mm}$ ) at the level of the inguinal canal prior to sclerotherapy to prevent reflux of sclerosant ${ }^{17}$.

A venogram was performed to assess the approximate volume of sclerosant required. The sclerosant (Sodium Tetradecyl Sulphate 3\%, Fibrovein, STD Pharmaceuticals, UK) was made into a foam using two Luer-lock syringes and a three-way stopcock using the standard Tessari method ${ }^{18} 19$. Following a test injection with contrast, approximately $2-6 \mathrm{mls}$ of sclerosant foam was injected slowly through the single end-hole 5-Fr / microcatheter under fluoroscopic guidance in the testicular vein. Coils $(6-10 \mathrm{~mm})$ were then placed at the approximate level of the mid sacro-iliac joints.

The catheter and sheath were then removed with haemostasis secured with direct pressure. The patients were discharged on the day of the procedure and asked to avoid sport and strenuous activity for 3 days, and sexual activity for 10 days(1). If patients developed post-procedure scrotal/testicular pain, they were advised to take non-steroidal anti-inflammatory drug (NSAID) analgesia if no contra-indications.

\section{Pain assessment}

Outcomes assessed were overall procedure success measured either by technical failure or by recurrence of clinically significant varicocele requiring further 
intervention. Pain was assessed in the form immediate post-procedural pain obtained from the pain score documented in the patient's Modified Early Warning Score (MEWS). A prospective validated pain impact questionnaire (PIQ 6, QualityMetric Inc., Lincoln, RI) ${ }^{20}$ was conducted pre and post treatment of the varicocele to assess for improvement in parameters such as pain severity, effect on work, enjoyment of life, interference with performing simple tasks, interference with leisure activities and finally impact on mood/feeling fed up.

Questionnaires were carried out by an independent interventional radiology nurse specialist both on the day of procedure and also at six weeks follow up over the phone. Numerical values were assigned based on severity/frequency of impact for the above individual parameters. Pain severity was scored 1-6 and the rest of the questions were scored 1-5. Total minimum score was 6 and the maximum possible score was 31 . Only patients with fully completed pre and post PIQ-6 questionnaires were included in the QoL element of the study. Patient data and questionnaire responses were anonymised and stored in a password protected file. Ethics approval was obtained from the local institution ethics committee (ref 2017-01-25 13-08-49). Statistical analysis was performed using SPSS (Chicago, IL, USA) software package. Matched pair student two tailed t test was used to compare mean scores pre and post treatment with $95 \%$ confidence intervals presented as $\mathrm{T}$ scores and their associated $\mathrm{P}$ values All tests of significance were 2-tailed, with $\mathrm{P}<0.050$ indicating statistical significance.

\section{Results}

Over a three-year period, 62 SVE procedures were performed for symptomatic varicocele. The mean age was 33.8 years (range 15-73). In this sample, 96\% $(n=59)$ cases were performed for a left sided varicocele. The most common clinical grade on examination was Grade II - palpable varicocele with no Valsalva but not visible. One quarter of patients had no clinical grade documented. The most common finding on ultrasound was medium/moderate sized varioccoele $(67 \%)$. There were no subclinical mild varicoceles included in this cohort as these patients were not offered and SVE and instead encouraged towards different management plan for their scrotal pain. Two patients had a failed procedure on two occasions requiring subsequent surgical ligation. There was one clinically significant recurrence at time of follow up, as demonstrated by recurrence of pain and associated increased swelling. This gives an overall success rate of $95 \%$ with a median follow up of 9 months. The average pain score on the post procedure MEWS observation sheath was low. The only complications were mild phlebitis (15\%) treated successful with non-steroidal anti-inflammatories and wound seroma (1\%). There were no major vascular or embolic complications. There was no incidence of hydrocoele at follow up. 


\section{Pain outcomes}

In total, 31 patients completed a PIQ-6 questionnaire pre- and post-procedure and were include in the second element of the study. There was a reduction of 8.774 in the mean total PIQ-6 score post procedure ( $\mathrm{t}$ score $-8.09, \mathrm{p}<0.0001)$ The breakdown of the individual PIQ-6 component mean scores pre- and post-SVE is presented below (Fig 1). All components showed a statistically significant reduction post treatment with SVE (Table 2). The most pronounced reductions were in the areas of pain frequency $[2.35(1.9,2.895 \% \mathrm{CI}), \mathrm{p}<0.05]$ and impact on leisure activities [2.0 $(1.64,2.3595 \% \mathrm{CI}), \mathrm{p}<0.05]$. All but one patient had a reduction in their mean total PIQ-6 score. The mean reported satisfaction rating with the procedure was 9.2/10.

\section{Discussion}

Testicular pain as an indication for intervention is often overlooked in the literature, as the primary focus of many studies to date has been on semen parameters and fertility. The main finding of the present study is that SVE is an effective and well tolerated procedure for symptomatic varicocele with low reported rates of periprocedural pain and just one recurrence at a median follow up of 9 months. Moreover, these results demonstrate that SVE may also significantly improve quality of life for this cohort of patients. In fact, in this series patients had a statistically significant improvement in each of the individual components of the PIQ-6 questionnaire.

The majority of studies looking at pain resolution as the primary outcome are in the field of surgical ligation. However most studies were retrospective with poor quality of evidence overall ${ }^{21}$. There have been few studies of SVE performed specifically for painful varicocele $\mathrm{e}^{22-24}$. Muthuveloe et al have published the largest series to date. Whilst the study was prospective it used only a Visual Analogue Scale (VAS). This has been found to have limitations in the field of pain medicine research and recommended to be used more as a screening tool ${ }^{25}$. Similar to the present study, Sheehan et al evaluated a more overall assessment of patient reported outcomes including impact on quality of life, and similar to their findings, our results demonstrate that SVE for symptomatic varicocele significantly improves quality of life for this cohort of patients. Recurrence rates were lower in our study and while there were a lower number of completed questionnaires, the fact that the questionnaires were carried out prospectively negates the retrospective and recall bias associated with the methodology of the previous study.

A combination of both detachable micro coil embolisation and sclerotherapy were used in this study. Different occlusion materials have been studied previously including acrylic glue ${ }^{26}$ and several types of coil material ${ }^{27}$. Whilst there is no difference in occlusion rates, recurrence rates are suggested to be lowest with acrylic glue ${ }^{27}$. A systematic review by Makris et al suggests that coil embolization has the highest rate of recurrence at $9.1 \%$ and additional sclerotherapy has no 
benefit ${ }^{27}$. In contradiction to this, our recurrence rate with both coil and sclerotherapy was low at $1.69 \%$. Whilst complications, including scrotal haematomas, epididymo-orchitis, recurrent testicular pain and testicular atrophy have been reported to occur in 3-5\% of patients undergoing antegrade sclerotherapy, we observed a low complication rate overall, with superficial phlebitis the most common complication. A more recent minimally invasive treatment for SVE is the use of endovascular laser ablation, which has been shown to be effective and safe, albeit in a low powered study ${ }^{28}$. The main advantage of this technique is it proposes to avoid the complications of migration, pulmonary embolus or stroke that can potentially be associated with coil embolization. Thankfully, none of these major complications were reported in our series.

One patient in this study had no improvement in his pain post SVE procedure. Of note, he had the highest PIQ-6 of all pre-procedure compared to a mean total score of 16. Perhaps this should have served as a sign that there were other factors at play such as neuropathic pain. It is important to try and identify such patients that will not do well post-SVE. One study suggest a spermatic cord block may be beneficial in pre-procedural assessment, in the setting of an inguinal hernia repair or vasectomy who present with a varicocele and pain ${ }^{29}$. Spermatic cord block with a long acting local anaesthetic agent may aid in diagnosing neuropathic pain in addition to providing guidance as to the type of intervention that may be warranted. For example, if a patient has a greater than $50 \%$ reduction in pain after spermatic cord block, microsurgical cord denervation may be an effective treatment option ${ }^{29}$.

Despite the reported benefits of SVE, including faster recovery, surgical repair to prevent venous reflux is a longer established technique and remains the most popular form of treatment for varicocele worldwide ${ }^{3}$. Open varicocelectomy (retroperitoneal high ligation, inguinal and sub-inguinal ligation), minimally invasive (laparoscopic/robotic varicocelectomy) or microsurgical varicocelectomy have all been described. However, there are a number of potentially severe complications that are exclusive to surgery including testicular ischaemia caused by failure to spare the testicular artery, hydrocoele formation (mostly from inadvertent ligation of the lymphatics during a high ligation) and nerve injury ${ }^{3}$. For the reasons above and also availability of technical expertise in our institution we have changed our practice over the last decade as urologists from offering surgery up front to reserving it as second line for those for whom SVE was not technically feasible. This change in approach in our institution is verified by the excellent recovery and success rates in this study.

There are a number of limitations of this study. Whilst the sample size is comparable to other studies in the literature in terms of the number of SVE procedures, only $50 \%$ of patients completed a full PIQ-6 questionnaire. This occurred for a combination of reasons including patient choice and incomplete or missing questionnaire data. Only patients with fully completed pre and post PIQ-6 questionnaires were included in the QoL element of the study. It was felt to be 
beneficial to include all patients who underwent SVE in the overall study as it maximised sample size in terms of technical outcomes. The authors accept that QoL outcomes cannot be applied to the whole cohort and indeed the statististical impact of a reduced response rate (ie the mean reduction in pain impact scores would be halved). However, we do provide robust data for 31 patients pre and post procedure and therefore we believe to be useful and representative of the wider population. There were no obvious differences between questionnaire responders and nonresponders in this study, negating a systemic reason or bias for non respsonse. Furthermore, recent literature suggests that chasing higher response rates in an attempt to reduce non-response bias is not worthwhile ${ }^{30}$. There was no duplex ultrasound follow up performed to assess for technical failure. The only patient who had a follow up ultrasound had a recurrence of symptoms. However, this is in keeping with the two similar studies mentioned above ${ }^{22} 23$. Puche-Sanz et al found that persistent venous reflux at follow was often present without clinical failure ${ }^{24}$. Finally, the study did not compare SVE to any other technique as it is now the mainstay first line treatment for symptomatic varicocele, but a randomised study comparing SVE to microsurgical varicocele repair including QoL data would be ideal.

\section{Conclusions}

In conclusion SVE for painful varicocele is associated with low peri-procedural pain, and patients treated in this manner achieve significant long-term improvement in both pain and quality of life as assessed using a prospective validated pain impact questionnaire. Moreover, rates of initial successful occlusion are high with minimal long-term recurrence, albeit with limited follow-up. In our experience, SVE offers an effective first line minimally invasive treatment for symptomatic varicocele and represents a viable first line alternative to the traditional surgical approach. 


\section{References}

1. Meacham RB, Townsend RR, Rademacher D, et al. The incidence of varicoceles in the general population when evaluated by physical examination, gray scale sonography and color Doppler sonography. J Urol 1994;151(6):1535-8. doi: 10.1016/s0022-5347(17)35295-3 [published Online First: 1994/06/01]

2. Ding H, Tian J, Du W, et al. Open non-microsurgical, laparoscopic or open microsurgical varicocelectomy for male infertility: a meta-analysis of randomized controlled trials. BJU Int 2012;110(10):1536-42. doi: 10.1111/j.1464-410X.2012.11093.x [published Online First: 2012/05/31]

3. Chan P. Management options of varicoceles. Indian J Urol 2011;27(1):6573. doi: 10.4103/0970-1591.78431

4. Thelen M, Weissbach L, Franken T. [The treatment of idiopathic varicoceles by transfemoral spiral occlusion of the left testicular vein (author's transl)]. RoFo : Fortschritte auf dem Gebiete der Rontgenstrahlen und der Nuklearmedizin 1979;131(1):24-9. doi: 10.1055/s-0029-1231369 [published Online First: 1979/07/01]

5. Kim J, Shin JH, Yoon HK, et al. Persistent or recurrent varicocele after failed varicocelectomy: Outcome in patients treated using percutaneous transcatheter embolization. Clinical Radiology 2012;67(4):359-65. doi: https://doi.org/10.1016/j.crad.2011.10.007

6. Iaccarino V, Venetucci P. Interventional radiology of male varicocele: current status. Cardiovascular and interventional radiology 2012;35(6):1263-80. doi: 10.1007/s00270-012-0350-z [published Online First: 2012/03/03]

7. Evers JH, Collins J, Clarke J. Surgery or embolisation for varicoceles in subfertile men. The Cochrane database of systematic reviews 2009(1):Cd000479. doi: 10.1002/14651858.CD000479.pub4 [published Online First: 2009/01/23]

8. Kroese AC, de Lange NM, Collins J, et al. Surgery or embolization for varicoceles in subfertile men. The Cochrane database of systematic reviews 2012;10:Cd000479. doi: 10.1002/14651858.CD000479.pub5 [published Online First: 2012/10/19]

9. The National Institute for Health and Care Excellence. Fertility problems: assessment and treatment 2004 [Available from:

https://www.nice.org.uk/guidance/cg156/chapter/Recommendations\#medical -and-surgical-management-of-male-factor-fertility-problems.

10. American Urological Association. Report on infertility and varicocele 2001 [cited 2020 3rd March]. Available from:

https://www.auanet.org/education/clinical-guidance/Varicocele-Archive.

11. Lomboy JR, Coward RM. The Varicocele: Clinical Presentation, Evaluation, and Surgical Management. Seminars in interventional radiology 2016;33(3):163-9. doi: 10.1055/s-0036-1586143 [published Online First: 2016/09/02] 
12. Peterson AC, Lance RS, Ruiz HE. Outcomes of varicocele ligation done for pain. J Urol 1998;159(5):1565-7. doi: 10.1097/00005392-199805000-00043 [published Online First: 1998/04/29]

13. Shoskes DA, Calixte N, Tadros N, et al. Validation of the Chronic Orchialgia Symptom Index for Men With Chronic Orchialgia/Chronic Scrotal Contents Pain. Urology 2018;119:39-43. doi: 10.1016/j.urology.2018.05.030 [published Online First: 2018/06/14]

14. Kavoussi PK, Costabile RA. Orchialgia and the chronic pelvic pain syndrome. World J Urol 2013;31(4):773-8. doi: 10.1007/s00345-013-1092-5 [published Online First: 2013/05/07]

15. Faculty of Radiologists RCoSI. Radiology Training in Ireland: Strategic Review 2016 [Available from: http://www.facultyofradiologists.com/images/uploads/2012/01/Strategic.Rev iew.FoR2016.FINAL .040816.pdf2021.

16. Dubin L, Amelar RD. Varicocele size and results of varicocelectomy in selected subfertile men with varicocele. Fertil Steril 1970;21(8):606-9. doi: 10.1016/s0015-0282(16)37684-1 [published Online First: 1970/08/01]

17. Flacke S, Schuster M, Kovacs A, et al. Embolization of varicocles: pretreatment sperm motility predicts later pregnancy in partners of infertile men. Radiology 2008;248(2):540-9. doi: 10.1148/radiol.2482071675 [published Online First: 2008/07/22]

18. Tessari L, Cavezzi A, Frullini A. Preliminary experience with a new sclerosing foam in the treatment of varicose veins. Dermatol Surg 2001;27(1):58-60. [published Online First: 2001/03/07]

19. Gandini R, Konda D, Reale CA, et al. Male varicocele: transcatheter foam sclerotherapy with sodium tetradecyl sulfate--outcome in 244 patients. Radiology 2008;246(2):612-8. doi: 10.1148/radiol.2462061295 [published Online First: 2008/01/30]

20. Becker J, Schwartz C, Saris-Baglama RN, et al. Using Item Response Theory (IRT) for Developing and Evaluating the Pain Impact Questionnaire (PIQ-6 ${ }^{\mathrm{TM}}$ ). Pain Medicine 2007;8(suppl_3):S129-S44. doi: 10.1111/j.15264637.2007.00377.x

21. Shridharani A, Lockwood G, Sandlow J. Varicocelectomy in the treatment of testicular pain: a review. Curr Opin Urol 2012;22(6):499-506. doi: 10.1097/MOU.0b013e328358f69f [published Online First: 2012/09/12]

22. Muthuveloe DW, During V, Ashdown D, et al. The effectiveness of varicocele embolisation for the treatment of varicocele related orchalgia. Springerplus 2015;4:392. doi: 10.1186/s40064-015-1177-2 [published Online First: 2015/08/08]

23. Sheehan M, Briody H, O'Neill DC, et al. Pain relief after varicocele embolization: The patient's perspective. J Med Imaging Radiat Oncol 2020 doi: 10.1111/1754-9485.13001 [published Online First: 2020/02/13]

24. Puche-Sanz I, Flores-Martín JF, Vázquez-Alonso F, et al. Primary treatment of painful varicocele through percutaneous retrograde embolization with fibred coils. Andrology 2014;2(5):716-20. doi: 10.1111/j.20472927.2014.00253.x

25. Saltychev M, Vastamäki H, Mattie R, et al. Psychometric Properties of the Pain Numeric Rating Scale When Applied to Multiple Body Regions among 
Professional Musicians. PLoS One 2016;11(9):e0161874. doi: 10.1371/journal.pone.0161874

26. Sze DY, Kao JS, Frisoli JK, et al. Persistent and recurrent postsurgical varicoceles: venographic anatomy and treatment with N-butyl cyanoacrylate embolization. J Vasc Interv Radiol 2008;19(4):539-45. doi: 10.1016/j.jvir.2007.11.009 [published Online First: 2008/04/01]

27. Makris GC, Efthymiou E, Little M, et al. Safety and effectiveness of the different types of embolic materials for the treatment of testicular varicoceles: a systematic review. Br J Radiol 2018;91(1088):20170445. doi: 10.1259/bjr.20170445 [published Online First: 2018/03/02]

28. Basile A, Motta A, Failla G, et al. Endovenous laser ablation of spermatic vein for the treatment of varicocele. Eur J Radiol Open 2017;4:129-31. doi: 10.1016/j.ejro.2017.09.001 [published Online First: 2017/10/17]

29. Long H, Bai W, Zhang X, et al. A Clinical Study on Microsurgical Denervation of Spermatic Cord for Refractory Chronic Orchialgia. Urologia Internationalis 2019;103(1):62-67. doi: 10.1159/000497281

30. Hendra R, Hill A. Rethinking Response Rates: New Evidence of Little Relationship Between Survey Response Rates and Nonresponse Bias. Eval Rev 2019;43(5):307-30. doi: 10.1177/0193841x18807719 [published Online First: $2018 / 12 / 26]$

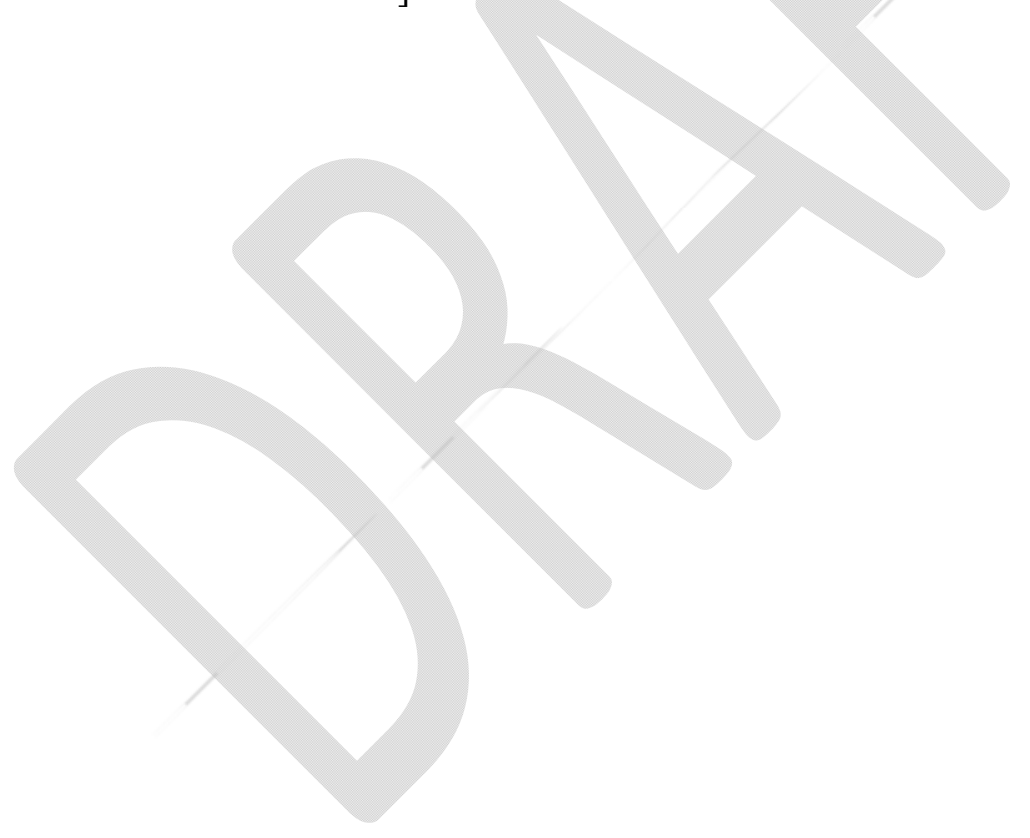


Figures and Tables

\begin{tabular}{|c|c|c|c|}
\hline Patient characteristics & & $\mathbf{n}$ & $\%$ \\
\hline Mean Age & $33.8 \mathrm{yrs}$ & (range 15-73) & \\
\hline Side & $\begin{array}{l}\text { Left } \\
\text { Right }\end{array}$ & $\begin{array}{c}59 \\
3\end{array}$ & $\begin{array}{c}96 \% \\
4 \%\end{array}$ \\
\hline Clinical grade & $\begin{array}{c}\text { Subclinical } \\
\text { Grade I } \\
\text { Grade II } \\
\text { Grade III } \\
\text { No grade given }\end{array}$ & $\begin{array}{c}0 \\
1 \\
35 \\
20 \\
16\end{array}$ & \begin{tabular}{|c|}
$0 \%$ \\
$1.6 \%$ \\
$56.4 \%$ \\
$32.2 \%$ \\
$25.8 \%$ \\
\end{tabular} \\
\hline Ultrasound findings & $\begin{array}{c}\text { Small/mild } \\
\text { Medium/moderate } \\
\text { Large/severe }\end{array}$ & $\begin{array}{c}0 \\
42 \\
20\end{array}$ & $\begin{array}{c}0 \% \\
67.7 \% \\
32.2 \%\end{array}$ \\
\hline
\end{tabular}

\begin{tabular}{|c|c|c|c|c|c|}
\hline $\begin{array}{l}\text { QoL impact pre } \\
\text { vs. post-SVE }\end{array}$ & $\begin{array}{l}\text { Mean score } \\
\text { reduction }\end{array}$ & SD & $95 \%$ CI & T score & $\begin{array}{c}\text { Significance } \\
\text { (2-tailed) }\end{array}$ \\
\hline Pain frequency & 2.35 & \pm 1.23 & $(1.9,2.8)$ & 10.69 & $<0.05$ \\
\hline $\begin{array}{l}\text { Interference with } \\
\text { work }\end{array}$ & 1.09 & \pm 1.3 & $(0.62,1.57)$ & 4.69 & $<0.05$ \\
\hline $\begin{array}{l}\text { Impact on } \\
\text { enjoyment of life }\end{array}$ & 1.23 & \pm 1.19 & $(0.89,1.76)$ & 6.17 & $<0.05$ \\
\hline $\begin{array}{l}\text { Make simple } \\
\text { tasks hard }\end{array}$ & 0.9 & \pm 1.3 & $(0.43,1.38)$ & 3.86 & $<0.05$ \\
\hline $\begin{array}{l}\text { Impact leisure } \\
\text { activities }\end{array}$ & 2.0 & \pm 0.97 & $(1.64,2.35)$ & 11.53 & $<0.05$ \\
\hline $\begin{array}{l}\text { Cause to feel fed } \\
\text { up }\end{array}$ & 1.16 & \pm 1.16 & $(0.73,1.59)$ & 5.59 & $<0.05$ \\
\hline
\end{tabular}

CI: confidence interval; QoL: quality of life; SD: standard deviation; SVE: spermatic vein embolization 